\title{
Some Remarks on the Generalized Scheme of Reduction to Absurdity and Generalized Hypothetical Syllogism in Fuzzy Logic
}

\author{
Katarzyna Miś and Michał Baczyński and Piotr Helbin \\ Institute of Mathematics, University of Silesia in Katowice, \\ 40-007 Katowice, Bankowa 14, Poland, \\ $\{$ kmis, michal . baczynski, piotr.helbin\}@us.edu.pl
}

\begin{abstract}
In this paper we investigate two generalizations, in fuzzy logic, of classical scheme of reduction to absurdity. We compare them with two possible generalizations of classical hypothetical syllogism (in fuzzy logic) and we show that generalized hypothetical syllogism is more general. We present new results concerning solutions of an inequality and an equation connected directly with generalization of scheme of reduction to absurdity in fuzzy logic.
\end{abstract}

Keywords: Generalized reduction to absurdity, generalized hypothetical syllogism, fuzzy implications, R-implications, t-norms, fuzzy negations

\section{Introduction}

There are many reasoning schemas (rules of inferences) in classical logic, like modus (ponendo) ponens, modus (tollendo) tollens, scheme of disjunctive reasoning, law of contraposition, etc. They are also applied in the terms of fuzzy logic. Namely, they are used in approximate reasoning and/or fuzzy control. Recently, we have investigated generalized hypothetical syllogism in fuzzy logic [2] (see also [7]). This notion can be introduced from a $T$-transitivity in the following way

$$
T(I(x, z), I(z, y)) \leq I(x, y), \quad x, y, z \in[0,1],
$$

where $T$ is a t-norm and $I$ a fuzzy implication. However, involving Zadeh's compositional rule of inference (CRI) [8] we can receive the following functional equation, satisfied for all $x, y \in[0,1]$,

$$
\sup _{z \in[0,1]}(T(I(x, z), I(z, y)))=I(x, y) .
$$

In this paper we investigate different scheme - reduction to absurdity (in Latin "reductio ad absurdum"). In general we can write it in fuzzy logic as follows

\begin{tabular}{lc} 
RULE: & IF $x$ is not $A$, THEN $y$ is $B$ \\
FACT: & $y$ is not $B$ \\
\hline CONCLUSION: & $x$ is $A$
\end{tabular}

where $A, B$ are fuzzy sets that represent some properties. Based on rules from the Boolean algebra and important investigations from [6], where some generalizations of classical schemes of reasoning were examined in fuzzy logic, we can write the following inequality, which corresponds with the reduction to absurdity,

$$
T(I(N(x), y), N(y)) \leq x, \quad x, y \in[0,1] .
$$

However, again using CRI it is possible to obtain the functional equation of the form

$$
\sup _{y \in[0,1]} T(I(N(x), y), N(y))=x, \quad x \in[0,1],
$$

where $N$ is a fuzzy negation, $T$ a t-norm and $I$ a fuzzy implication.

The main goal of this article is to compare written above inequalities and equations for generalized hypothetical syllogism and generalized scheme of reduction to absurdity in fuzzy logic. Moreover, we give some new results concerning particular solutions of (RA) and (GRA).

The paper is organised as follows. Section 2 contains some important facts and definitions used in the sequel, while in Section 3 we present some properties regarding (RA) and some solutions of (RA) and (GRA) for several families of fuzzy implications. We also present some new results concerning (GHS).

\section{Preliminaries}

To make this work more self-contained, we place some of basic definitions concerning fuzzy connectives here. Note that the family of all increasing bijections $\varphi:[0,1] \rightarrow[0,1]$ will be denoted by the symbol $\Phi$.

Definition 2.1 (see $[3,5]$ ). A function $T:[0,1]^{2} \rightarrow$ $[0,1]$ is called a triangular norm (t-norm in short), 
if it satisfies, for all $x, y, z \in[0,1]$, the following conditions:

(T1) $T(x, y)=T(y, x)$,

(T2) $T(x, T(y, z))=T(T(x, y), z)$,

(T3) $T(x, y) \leq T(x, z)$ for $y \leq z$, i.e., $T(x, \cdot)$ is nondecreasing,

(T4) $T(x, 1)=x$.

Definition 2.2 (see [5]). A function $S:[0,1]^{2} \rightarrow[0,1]$ is called a triangular conorm (t-conorm in short), if it satisfies, for all $x, y, z \in[0,1]$, the following conditions:

(S1) $S(x, y)=S(y, x)$,

(S2) $S(x, S(y, z))=S(S(x, y), z)$,

(S3) $S(x, y) \leq S(x, z)$ for $y \leq z$, i.e., $S(x, \cdot)$ is nondecreasing,

(S4) $S(x, 0)=x$.

Example 2.3. 1. The Łukasiewicz t-norm,

$$
T_{\mathbf{L K}}(x, y)=\max (x+y-1,0), \quad x, y \in[0,1] .
$$

2. The Łukasiewicz t-conorm,

$$
S_{\mathbf{L K}}(x, y)=\min (x+y, 1), \quad x, y \in[0,1] .
$$

Definition 2.4 (see $[1,5]$ ). A non-increasing function $N:[0,1] \rightarrow[0,1]$ is called a fuzzy negation, if $N(0)=1, N(1)=0$. Moreover, a fuzzy negation $N$ is called

(i) strict, if it is strictly decreasing and continuous,

(ii) strong, if it is an involution, i.e., $N(N(x))=x$ for all $x \in[0,1]$.

Example 2.5. 1. The classical negation $N_{\mathbf{C}}$ is given by $N_{\mathbf{C}}(x)=1-x$, for $x \in[0,1]$.

2. The least negation $N_{\mathbf{D}_{\mathbf{1}}}$ is given by

$$
N_{\mathbf{D}_{1}}(x)=\left\{\begin{array}{ll}
1, & x=0, \\
0, & x>0,
\end{array} \quad x \in[0,1]\right.
$$

Definition 2.6 ([1, Definition 2.3.14]). Let $T$ be a tnorm and $N$ a fuzzy negation. We say that a pair $(T, N)$ satisfies the law of contradiction if

$$
T(x, N(x))=0, \quad x \in[0,1] .
$$

Now, we recall the definition and some important properties of fuzzy implications.
Definition 2.7 (see $[1,3]$ ). A function $I:[0,1]^{2} \rightarrow$ $[0,1]$ is called a fuzzy implication, if it satisfies the following conditions.

(I1) $I$ is non-increasing with respect to the first variable,

(I2) $I$ is non-decreasing with respect to the second variable,

(I3) $I(0,0)=I(1,1)=1$ and $I(1,0)=0$.

The family of all fuzzy implications will be denoted by $\mathcal{F} \mathcal{I}$.

Definition 2.8 (see [1]). We say that a fuzzy implication $I$ satisfies

(i) the identity principle, if

$$
I(x, x)=1, \quad x \in[0,1],
$$

(ii) the left neutrality property, if

$$
I(1, y)=y, \quad y \in[0,1],
$$

(iii) the ordering property, if

$$
x \leq y \Longleftrightarrow I(x, y)=1, \quad x, y \in[0,1] .
$$

(iv) law of contraposition with respect to $N$, if

$$
I(x, y)=I(N(y), N(x)), \quad x, y \in[0,1],
$$

(v) law of left contraposition with respect to $N$, if

$$
I(N(x), y)=I(N(y), x), \quad x, y \in[0,1],
$$

(vi) law of right contraposition with respect to $N$, if

$$
I(x, N(y))=I(y, N(x)), \quad x, y \in[0,1] . \quad(\mathrm{R}-\mathrm{CP})
$$

Definition 2.9 ([1, Definition 1.4.15]). Let $I$ be a fuzzy implication. A function $N_{I}:[0,1] \rightarrow[0,1]$ given by

$$
N_{I}(x)=I(x, 0), \quad x \in[0,1],
$$

is called the natural negation of $I$.

Let us also recall definitions of two families of fuzzy implications.

Definition 2.10 ([1, Definition 2.5.1]). A function $I:[0,1]^{2} \rightarrow[0,1]$ is called a residual implication $(\mathrm{R}-$ implication for short) if there exists a t-norm $T$ such that

$$
I(x, y)=\sup \{t \in[0,1] \mid T(x, t) \leq y\}, \quad x, y \in[0,1] .
$$

If $I$ is generated from a t-norm $T$, then it will be denoted by $I_{T}$. 
Definition 2.11 ([1, Definition 2.4.1]). A function $I:[0,1]^{2} \rightarrow[0,1]$ is called an $(S, N)$-implication, if there exist a t-conorm $S$ and a fuzzy negation $N$ such that

$$
I(x, y)=S(N(x), y), \quad x, y \in[0,1] .
$$

If $I$ is generated from a t-conorm $S$ and a fuzzy negation $N$, then it will be denoted by $I_{S, N}$.

\section{Properties of (RA) and (GRA)}

Let us start with some general properties of triplets $(T, I, N)$ satisfying (RA).

Proposition 3.1. Let $I \in \mathcal{F} \mathcal{I}, T$ be a t-norm and $N$ be a fuzzy negation. Next, let the triplet $(T, I, N)$ satisfies (RA).

1. If I satisfies $(\mathrm{NP})$, then $(T, N)$ satisfies (LC).

2. If $N_{I}$ is injective, then $N_{I}^{-1} \leq N$.

3. If I satisfies (NP), then $N \leq N_{T}$.

4. If $N \neq N_{\mathbf{D}_{1}}$ and I satisfies (NP), then $T$ has zero-divisors $(a \in(0,1)$ is a zero-divisor if there exists $b \in(0,1)$ such that $T(a, b)=0)$.

5. If $N_{I}(x) \neq 0, x \in[0,1), T$ is continuous and $I$ satisfies (NP), then there exists $\varphi \in \Phi$ such that

$$
\begin{aligned}
T(x, y) & =\left(T_{\mathbf{L K}}\right)_{\varphi}(x, y) \\
& =\varphi^{-1}(\max (0, \varphi(x)+\varphi(y)-1)),
\end{aligned}
$$

for all $x, y \in[0,1]$.

6. If $I_{1} \in \mathcal{F I}, T_{2}$ is a t-norm such that $T_{2} \leq T$ and $I_{1} \leq I$, then $\left(T_{2}, I_{1}\right)$ satisfies (RA).

Proof. 1. It is enough to take $x=0$. Hence we have $0 \leq T(N(y), I(1, y))=T(N(y), y) \leq 0$, for all $y \in$ $[0,1]$.

2. If we take $y=0$ and we assume $N_{I}$ is injective, then for every $x \in[0,1]$ we obtain

$$
\begin{aligned}
T(1, I(N(x), 0)) & \leq x, \\
I(N(x), 0) & \leq x, \\
N_{I}(N(x)) & \leq x, \\
N(x) & \geq N_{I}^{-1}(x) .
\end{aligned}
$$

3. It is immediate from the point 1 . and from the formula of $N_{T}$ (negation induced by $T$ ) given by

$$
N_{T}(x)=\sup \{y \in[0,1] \mid T(x, y)=0\},
$$

for $x \in[0,1]$.
4. If $N \neq N_{\mathrm{D}_{1}}$, then there exists $x \in(0,1)$ such that $N(x)>0$. Hence from the point 1. $T$ has zero-divisors because $(T, N)$ satisfies (LC).

5. If $N_{I}(x)>0$ for $x \in[0,1)$, then every such $x$ is a zero-divisor of $T$. Moreover, the only continuous t-norm such the set of zero-divisors is $(0,1)$ is $T=\left(T_{\mathbf{L K}}\right)_{\varphi}$, for some $\varphi \in \Phi$ (see [5, Remark 2.4, Proposition 5.10]).

6. It is straightforward from the following inequalities,

$$
\begin{aligned}
T_{2}\left(N(y), I_{1}(N(x), y)\right) & \leq T_{2}(N(y), I(N(x), y)) \\
& \leq T(N(y), I(N(x), y)) \\
& \leq x,
\end{aligned}
$$

satisfied for all $x, y \in[0,1]$.

Now, we recall one result concerning triplets $(T, I, N)$ satisfying the (RA) but with some strong assumptions regarding $T$ and $N$.

Theorem 3.2 ([6, Proposition 6.3]). Let $T$ be a continuous $t$-norm, $N$ a strong negation and let $I \in \mathcal{F} \mathcal{I}$ satisfy (NP). Then the following statements are equivalent.

(i) The triplet $(T, I, N)$ satisfies (RA).

(ii) there exists $\varphi \in \Phi$ such that $T=\left(T_{\mathbf{L K}}\right)_{\varphi}, N \leq$ $\left(N_{\mathbf{C}}\right)_{\varphi}$ and

$\varphi(I(x, y)) \leq 1-\varphi(N(y))+\varphi(N(x)), x, y \in[0,1]$.

Of course we can find some solutions of (RA), where $T$ is non-continuous.

Example 3.3. Let us consider the drastic (noncontinuous) t-norm given by

$$
T_{\mathbf{D}}(x, y)= \begin{cases}0, & x, y \in[0,1), \\ \min (x, y), & \text { otherwise, }\end{cases}
$$

and the Gödel implication given by

$$
I_{\mathbf{G D}}(x, y)= \begin{cases}1, & x \leq y \\ y, & x>y\end{cases}
$$

Then it can be quite easily verified that the triplet $\left(T_{\mathbf{D}}, I_{\mathbf{G D}}, N_{\mathbf{C}}\right)$ satisfies $(\mathrm{RA})$.

It is obvious that if a triplet $(T, I, N)$ satisfies (GRA), then such triplet satisfies also (RA). Similarly, if a pair $(T, I)$ satisfies $(\mathrm{GHS})$, then $(T, I)$ satisfies also (HS). In general, without additional assumptions, the opposite implications are not true.

Example 3.4. The triplet $\left(T_{\mathbf{D}}, I_{\mathbf{G D}}, N_{\mathbf{C}}\right)$ satisfies (RA) and does not satisfy (GRA). Furthermore, 
the pair $\left(T_{\mathbf{D}}, I_{\mathbf{R C}}\right)$ satisfies (HS) and does not satisfy (GHS), where $I_{\mathbf{R C}}$ is the Reichenbach implication given by the formula $I_{\mathbf{R C}}(x, y)=1-x+x y$, for $x, y \in[0,1]$.

The next results show some sufficient conditions to satisfy (GHS) and (GRA).

Proposition 3.5. Let $T$ be a t-norm and $I \in \mathcal{F} \mathcal{I}$. If the pair $(T, I)$ satisfies (HS) and I satisfies (IP), then $(T, I)$ satisfies (GHS).

Proof. Let $x, y \in[0,1]$. From one side, from (HS) and monotonicity of supremum we have

$$
\sup _{z \in[0,1]}(T(I(x, z), I(z, y))) \leq \sup _{z \in[0,1]} I(x, y)=I(x, y) .
$$

On the other side,

$$
\begin{aligned}
\sup _{z \in[0,1]}(T(I(x, z), I(z, y))) & \geq T(I(x, y), I(y, y)) \\
& =T(I(x, y), 1)=I(x, y) .
\end{aligned}
$$

Therefore (GHS) is true for the pair $(T, I)$.

However, the above condition is not necessary.

Example 3.6. Let $I_{\text {KD }}$ be the Kleene-Dienes implication given by

$$
I_{\mathbf{K D}}(x, y)=\max (1-x, y), \quad x, y \in[0,1] .
$$

Then the pair $\left(T_{\mathbf{D}}, I_{\mathbf{K D}}\right)$ satisfies (HS) although $I_{\mathbf{K D}}$ does not satisfy (IP). Moreover, this pair satisfies (GHS). Indeed, if $x=0$, then for all $y \in[0,1]$

$$
\begin{aligned}
\sup _{z \in[0,1]} & T_{\mathbf{D}}\left(I_{\mathbf{K D}}(0, z), I_{\mathbf{K D}}(z, y)\right) \\
& =\sup _{z \in[0,1]} T_{\mathbf{D}}(1, \max (1-z, y)) \\
& =\sup _{z \in[0,1]} \max (1-z, y)=1=I_{\mathbf{K D}}(x, y),
\end{aligned}
$$

and similarly, if $y=1$, then for all $z \in[0,1]$ we have

$$
\begin{aligned}
\sup _{z \in[0,1]} & T_{\mathbf{D}}\left(I_{\mathbf{K D}}(x, z), I_{\mathbf{K D}}(z, 1)\right) \\
= & \sup _{z \in[0,1]} T_{\mathbf{D}}(\max (1-x, z), 1) \\
\quad= & \sup _{z \in[0,1]} \max (1-x, z)=1=I_{\mathbf{K D}}(x, y) .
\end{aligned}
$$

Next, from the definition of the drastic t-norm $T_{\mathbf{D}}$, for every $x \in(0,1], y \in[0,1)$ and $z \in(0,1)$ we have

$$
T_{\mathbf{D}}\left(I_{\mathbf{K D}}(x, z), I_{\mathbf{K D}}(z, y)\right)=0,
$$

thus when $x \in(0,1]$ and $y \in[0,1)$ we have

$$
\begin{aligned}
\sup _{z \in[0,1]} T_{\mathbf{D}}\left(I_{\mathbf{K D}}(x, z), I_{\mathbf{K D}}(z, y)\right) & \\
= & \max \left(T_{\mathbf{D}}\left(I_{\mathbf{K D}}(x, 0), I_{\mathbf{K D}}(0, y)\right),\right. \\
& \left.T_{\mathbf{D}}\left(I_{\mathbf{K D}}(x, 1), I_{\mathbf{K D}}(1, y)\right)\right) \\
= & \max (1-x, y)=I_{\mathbf{K D}}(x, y) .
\end{aligned}
$$

Therefore the pair $\left(T_{\mathbf{D}}, I_{\mathbf{K D}}\right)$ satisfies (GHS).
Since each R-implication satisfies (IP) (see e.g. [1, Theorem 2.5.4]), the following fact is true for all R-implications.

Corollary 3.7. For a t-norm $T$ the following statements are equivalent.

(i) The pair $\left(T, I_{T}\right)$ satisfies (HS).

(ii) The pair $\left(T, I_{T}\right)$ satisfies (GHS).

Now, let us return to (RA) and (GRA).

Proposition 3.8. Let $T$ be a t-norm, $N$ be a strict negation and $I \in \mathcal{F} \mathcal{I}$. If the triplet $\left(T, I, N^{-1}\right)$ satisfies (RA) and $N_{I} \circ N^{-1}=i d$ (i.e., $\left.N_{I}=N\right)$, then the triplet $\left(T, I, N^{-1}\right)=\left(T, I, N_{I}^{-1}\right)$ satisfies (GRA).

Proof. Let $x \in[0,1]$. On the one hand, from (RA) and monotonicity of supremum we have

$$
\sup _{y \in[0,1]} T\left(I\left(N^{-1}(x), y\right), N^{-1}(y)\right) \leq \sup _{y \in[0,1]} x=x .
$$

On the other hand, from our assumption we obtain

$$
\begin{aligned}
\sup _{y \in[0,1]} T\left(I\left(N^{-1}(x), y\right), N^{-1}(y)\right) \\
\quad \geq T\left(I\left(N^{-1}(x), 0\right), N^{-1}(0)\right) \\
\quad=T\left(I\left(N^{-1}(x), 0\right), 1\right)=N_{I} \circ N(x)=x .
\end{aligned}
$$

Therefore (GRA) is true for the triplet $\left(T, I, N^{-1}\right)$.

Example 3.9. It can be easily checked that the triplet $\left(T, I, N_{I}\right)$, where $T=T_{\mathbf{D}}$ and

$$
I(x, y)=\max \left(1-x^{2}, y\right), \quad x, y \in[0,1],
$$

satisfies (RA). From the above result we know that this triplet satisfies also (GRA).

Proposition 3.10. Let $T$ be a t-norm, $I \in \mathcal{F} \mathcal{I}$ and let $N_{I}$ be a strong negation.

1. If the pair $(T, I)$ satisfies (GHS), then the triplet $\left(T, I, N_{I}\right)$ satisfies (GRA).

2. If the pair $(T, I)$ satisfies (HS), then the triplet $\left(T, I, N_{I}\right)$ satisfies (RA).

Proof. 1. Let us take $y=0$ and substitute $N_{I}(x)$ instead of $x$ in (GHS). Then we have the following equations

$$
\begin{aligned}
& \sup _{z \in[0,1]} T\left(I\left(N_{I}(x), z\right), I(z, 0)\right)=I\left(N_{I}(x), 0\right), \\
& \sup _{z \in[0,1]} T\left(I\left(N_{I}(x), z\right), N_{I}(z)\right)=N_{I}\left(N_{I}(x)\right), \\
& \sup _{z \in[0,1]} T\left(I\left(N_{I}(x), z\right), N_{I}(z)\right)=x,
\end{aligned}
$$

which means that the triplet $\left(T, I, N_{I}\right)$ satisfies (GRA). 
2. This proof is similar to that above.

Therefore, in some cases we can apply the following theorem valid for R-implications.

Theorem 3.11 ([2, Theorem 4.12]). Let $T^{*}$ be a $t$ norm and $T$ be a left-continuous t-norm. Then the following statements are equivalent.

(i) The pair $\left(T^{*}, I_{T}\right)$ satisfies (GHS).

(ii) $T^{*} \leq T$

Corollary 3.12. Let $T^{*}$ be a t-norm, $T$ be a leftcontinuous t-norm and $N_{I_{T}}$ be a strong negation. Then the following statements are equivalent.

(i) The triplet $\left(T^{*}, I_{T}, N_{I_{T}}\right)$ satisfies (GRA).

(ii) $T^{*} \leq T$.

Proof. $(i) \Longrightarrow(i i)$ Since $T$ is a left-continuous tnorm, the following equivalence is true (see $[1$, Proposition 2.5.2]), for all $x, y \in[0,1]$,

$$
T(x, y) \leq T(x, y) \Longleftrightarrow I_{T}(x, T(x, y)) \geq y .
$$

Let us recall that if $N=N_{I_{T}}$ is a strong negation, then $I_{T}$ satisfies (L-CP) with $N$ (see [1, Proposition 2.5.28]). Hence, for arbitrary fixed $x, y \in[0,1]$, we have

$$
\begin{aligned}
T(x, y) & =\sup _{z \in[0,1]} T^{*}\left(I_{T}(N(T(x, y)), z), N(z)\right) \\
& =\sup _{z \in[0,1]} T^{*}\left(I_{T}(N(z), T(x, y)), N(z)\right) \\
& \geq T^{*}\left(I_{T}\left(N\left(N^{-1}(x)\right), T(x, y)\right), N\left(N^{-1}(x)\right)\right) \\
& =T^{*}\left(I_{T}(x, T(x, y)), x\right) \geq T^{*}(x, y) .
\end{aligned}
$$

$($ ii $) \Longrightarrow(i)$ If $T^{*} \leq T$, then from Theorem 3.11 we know that the pair $\left(T^{*}, I_{T}\right)$ satisfies (GHS). Thus in virtue of Proposition 3.10 we obtain that the triplet $\left(T^{*}, I_{T}, N_{I_{T}}\right)$ satisfies (GRA).

Moreover, we have also the following fact.

Proposition 3.13. For a t-norm $T$ the following statements are equivalent.

(i) The pair $\left(T, I_{T}\right)$ satisfies (HS).

(ii) $T$ is left-continuous.

Proof. $(i) \Longrightarrow($ ii $)$ Suppose that the pair $\left(T, I_{T}\right)$ satisfies (HS) and $T$ is not left-continuous. Then there exist $x, y, z \in[0,1]$ such that $I_{T}(x, y) \geq z$ and $T(x, z)>y$ (see [1, Proposition 2.5.2]). Hence

$$
\begin{aligned}
y & <T(x, z) \leq T\left(x, I_{T}(x, y)\right) \\
& =T\left(I_{T}(1, x), I_{T}(x, y)\right) \leq I_{T}(1, y) \\
& =y
\end{aligned}
$$

a contradiction.

$($ ii $) \Longrightarrow(i)$ Note that (HS) is nothing else but the $T$ transitivity - the property satisfied for pairs $\left(T, I_{T}\right)$, where $T$ is a left-continuous t-norm (see [4, Proposition 1.6]).

Now we can formulate the following corollary.

Corollary 3.14. Let $T$ be a t-norm. If $I_{T}$ satisfies (L-CP) with a negation $N$, then the following statements are equivalent.

(i) The triplet $\left(T, I_{T}, N\right)$ satisfies (RA).

(ii) $T$ is left-continuous.

Proof. $(i) \Longrightarrow(i i)$ This part of the proof is similar to the proof of Proposition 3.13. Suppose that $I_{T}$ is not left-continuous. Then there exist $x, y, z \in[0,1]$ such that $I_{T}(y, x) \geq z$ and $T(y, z)>x$. Note that if $I_{T}$ satisfies (L-CP) with a negation $N$, then $N=N_{I_{T}}$ is strong ([1, Proposition 2.5.26]), and therefore $y=$ $N\left(y_{0}\right)$ for some $y_{0} \in[0,1]$. Hence

$$
\begin{aligned}
x & <T(y, z) \leq T(y, I(y, x)) \\
& =T\left(N\left(y_{0}\right), I_{T}\left(N\left(y_{0}\right), x\right)\right) \\
& =T\left(N\left(y_{0}\right), I_{T}\left(N(x), y_{0}\right)\right) \\
& \leq x
\end{aligned}
$$

a contradiction.

$($ ii $) \Longrightarrow(i)$ If $T$ is left-continuous and $I_{T}$ satisfies (L-CP) with $N$, then again from [1, Proposition 2.5.2] we have

$$
\begin{aligned}
& I_{T}(N(y), x) \geq I_{T}(N(x), y) \Longleftrightarrow \\
& T\left(N(y), I_{T}(N(x), y)\right) \leq x,
\end{aligned}
$$

for all $x, y \in[0,1]$.

Remark 3.15. We know that if $I_{T}$ satisfies (L-CP) with a negation $N$, then $N=N_{I_{T}}$ is strong (see [1, Proposition 2.5.26]). However it is not equivalent with left-continuity of $T$. Indeed, the Fodor implication given by

$$
I_{\mathbf{F D}}(x, y)= \begin{cases}1, & x \leq y \\ \max (1-x, y), & x>y\end{cases}
$$


which satisfies (L-CP) with $N_{\mathbf{C}}$ can be generated from the non left-continuous t-norm $T_{n M^{*}}$ given by

$$
T_{\mathbf{n M}^{*}}(x, y)= \begin{cases}0, & x+y<1 \\ \min (x, y), & \text { otherwise }\end{cases}
$$

One of sufficient conditions in such cases for $T$ to be left-continuous can be satisfying (RA) by the triplet $\left(T, I_{T}, N_{I_{T}}\right)$.

Remark 3.16. Assumptions of having left-continuous t-norm and strong negation $N$ were crucial in previous results. Note that for such fuzzy negation $(S, N)$ implication $I_{S, N}$ satisfies (L-CP) with $N$ (see $[1$, Proposition 2.4.3]). However, if we consider such $(S, N)$-implications, which are also R-implications generated from a left-continuous t-norm, we obtain only $I_{S, N}=\left(I_{\mathbf{L K}}\right)_{\varphi}$, for $\varphi \in \Phi$, where $I_{\mathbf{L K}}$ is the Eukasiewicz implication given by the formula $I_{\mathbf{L K}}(x, y)=\min (1-x+y, 1)$, for all $x, y \in[0,1]$.

Example 3.17. Despite this, we can find $(S, N)$ implications (which are not R-implications at the same time) satisfying (RA), for example the triplets $\left(T_{\mathbf{L K}}, I_{\mathbf{R C}}, N_{\mathbf{C}}\right)$ and $\left(T_{\mathbf{D}}, I_{\mathbf{R C}}, N_{\mathbf{C}}\right)$ satisfy $(\mathrm{RA})$.

Theorem 3.18. Let $T$ be a continuous t-norm, $S$ be a t-conorm, $N$ be a strong negation and let $I$ be an $(S, N)$-implication. If the triplet $(T, I, N)$ satisfies (GRA), then $T=\left(T_{\mathbf{L K}}\right)_{\varphi}$ and $N \leq\left(N_{\mathbf{C}}\right)_{\varphi}$, for some $\varphi \in \Phi$. Moreover, if $T=\left(T_{\mathbf{L K}}\right)_{\varphi}, N \leq\left(N_{\mathbf{C}}\right)_{\varphi}$ and $S \leq\left(S_{\mathbf{L K}}\right)_{\varphi}$, for some $\varphi \in \Phi$, then the triplet $(T, I, N)$ satisfies (GRA).

Proof. Assume that the triplet $(T, I, N)$ satisfies (GRA). From Theorem 3.2 we know that $T=$ $\left(T_{\mathbf{L K}}\right)_{\varphi}$ and $N \leq\left(N_{\mathbf{C}}\right)_{\varphi}$, for some $\varphi \in \Phi$.

Assume now that $T=\left(T_{\mathbf{L K}}\right)_{\varphi}, N \leq\left(N_{\mathbf{C}}\right)_{\varphi}$ and $S \leq$ $\left(S_{\mathbf{L K}}\right)_{\varphi}$, for some $\varphi \in \Phi$. Thus, for $x, y \in[0,1]$, we obtain

$$
\begin{aligned}
& T(N(y), I(N(x), y))=\left(T_{\mathbf{L K}}\right)_{\varphi}(N(y), S(x, y)) \\
& =\varphi^{-1}(\max (\varphi(N(y))+\varphi(S(x, y))-1,0)) \\
& \leq \varphi^{-1}\left(\max \left(\varphi\left(\left(N_{\mathbf{C}}\right)_{\varphi}(y)\right)+\varphi\left(\left(S_{\mathbf{L K}}\right)_{\varphi}(x, y)\right)-1,0\right)\right) \\
& \leq \varphi^{-1}(\max (-\varphi(y)+\min (\varphi(x)+\varphi(y), 1), 0)) \\
& \leq \varphi^{-1}(\max (\min (\varphi(x), 1-\varphi(y)), 0)) \\
& \leq \varphi^{-1}(\varphi(x))=x
\end{aligned}
$$

which proves that the triplet $(T, I, N)$ satisfies (RA). However, $N_{I}=N$ and $N$ is the strong negation. From Proposition 3.8 we obtain the thesis.

Let us finish with the following example which illustrates the last theorem.
Example 3.19. Consider the following functions

$$
\begin{aligned}
I(x, y) & =\max (1-x, y), \quad x, y \in[0,1], \\
T(x, y) & =\sqrt{\max \left(x^{2}+y^{2}-1,0\right)}, \quad x, y \in[0,1], \\
N_{1}(x) & =\sqrt{1-x^{2}}, \quad x \in[0,1] .
\end{aligned}
$$

Thus, $I$ is the Kleene-Dienes implication (it is an $(S, N)$-implication, cf. [1, Table 2.4]), $T=\left(T_{\mathbf{L K}}\right)_{\varphi}$ and $N_{1}=\left(N_{\mathbf{C}}\right)_{\varphi}$, where $\varphi(x)=x^{2}$, for all $x \in[0,1]$. From the above theorem the triplet $(T, I, N)$ satisfies (GRA), where $N=N_{C} \leq\left(N_{\mathbf{C}}\right)_{\varphi}$.

\section{Conclusions}

We have investigated the scheme of reduction to absurdity (GRA) and generalized hypothetical syllogism (GHS). We presented some similar results for both of them. Also we shown that in some cases (GHS) is more general. Moreover, we presented conditions when (RA) is equivalent to (GRA).

\section{Acknowledgement}

M. Baczyński and K. Miś acknowledge the support of the National Science Centre, Poland, under Grant No. 2015/19/B/ST6/03259.

\section{References}

[1] M. Baczyński, B. Jayaram, Fuzzy Implications, Vol. 231 of Studies in Fuzziness and Soft Computing, Springer, Berlin Heidelberg, 2008.

[2] M. Baczyński, K. Miś, Selected properties of generalized hypothetical syllogism including the case of R-implications, in: M. J. et al. (eds) (Ed.), Information Processing and Management of Uncertainty in Knowledge-Based Systems. Theory and Foundations, Vol. 853 of Communications in Computer and Information Science, Springer, Cham, 2018, pp. 673-684.

[3] J. Fodor, M. Roubens, Fuzzy Preference Modelling and Multicriteria Decision Support, Kluwer Academic Publishers, Dordrecht, 1994.

[4] S. Gottwald, Fuzzy Sets and Fuzzy Logic. The Foundations of Application - from a Mathematical Point of View, Vieweg+Teubner Verlag, Braunschweig/Wiesbaden, 1993.

[5] E. P. Klement, R. Mesiar, E. Pap, Triangular Norms, Kluwer Academic Publishers, Dordrecht, 2000 .

[6] E. Trillas, C. Alsina, E. Renedo, On some schemes of reasoning in fuzzy logic, New Math. Nat. Comput 7 (3) (2011) 433-451. 
[7] N. R. Vemuri, Investigations of fuzzy implications satisfying generalized hypothetical syllogism, Fuzzy Sets and Systems 323 (2017) 117-137.

[8] L. A. Zadeh, Outline of a new approach to the analysis of complex systems and decision processes, IEEE Trans. on Syst. Man and Cyber. 3 (1973) 2844. 